\title{
Chromosome Loops, Insulators, and Histone Methylation: New Insights into Regulation of Imprinting in Clusters
}

\author{
W. Reik, ${ }^{* \dagger}$ A. Murrell,${ }^{\dagger}$ A. Lewis,${ }^{\dagger}$ K. Mitsuya,${ }^{\dagger}$ D. Umlauf,${ }^{\S}$ W. Dean,${ }^{\dagger}$ \\ M. HigGins, ${ }^{\prime \prime}$ AND R FEIL ${ }^{\S}$ \\ † Laboratory of Developmental Genetics and Imprinting, The Babraham Institute, Cambridge \\ CB2 4AT, United Kingdom; ${ }^{*}$ The Wellcome Trust Sanger Institute, Wellcome Trust Genome Campus, \\ Hinxton, Cambridge CB10 1SA, United Kingdom; ' Institute of Molecular Genetics, CNRS, \\ UMR-5535, and University of Montpellier-II, 34293 Montpellier cedex, France; \\ "Department of Cancer Genetics, Roswell Park Cancer Institute, Buffalo, New York 14263
}

Imprinted genes in mammals are organized into clusters in which genes share regulatory elements. Igf2 and H19 are separated by $100 \mathrm{~kb}$ (kilobases) of DNA, and both genes use enhancers that are located distal to H19. Alternate access to the enhancers by the two genes is in part regulated by a CTCF-dependent insulator located upstream of $H 19$. We find that differentially methylated regions in both genes interact physically over the $100-\mathrm{kb}$ distance. These interactions are epigenetically regulated and partition maternal and paternal chromatin into distinct loops. This creates a simple epigenetic switch for Igf2 whereby it moves between an active and a silent chromatin domain. In the adjacent Kcnqlot1 cluster, by contrast, a noncoding RNA gene is flanked by several silent genes, which are marked by repressive histone modifications. Histone methylation is targeted directly or indirectly to the region by the noncoding RNA and is maintained in the absence of DNA methylation. We propose that imprinting regulation in clusters falls into different categories. The "insulator-loop" model may also be applicable to Dlk1/Gtl2 and Rasgrfl. The "noncoding RNA" model is likely to be applicable to Igf $2 r / A i r$, to imprinted X-inactivation, and to other maternally imprinted loci.

Genomic imprinting was discovered 20 years ago (McGrath and Solter 1984; Surani et al. 1984). The realization of developmental nonequivalence of the two parental genomes (the maternal and the paternal one) was quickly followed by the insight that epigenetic mechanisms such as DNA methylation are key to this parental chromosomal identity (Sasaki et al. 1992; Brandeis et al. 1993; Stoger et al. 1993; Ferguson-Smith and Surani 2001; Reik and Walter 2001; Sleutels and Barlow 2002; Verona et al. 2003; Delaval and Feil 2004). At the same time, the "genetic conflict" hypothesis of evolution of imprinting was conceived (Haig and Westoby 1989), followed by the identification of the first imprinted genes in mice and humans (Barlow et al. 1991; Bartolomei et al. 1991;

\footnotetext{
*To whom correspondence should be addressed. E-mail: wolf.reik@bbsrc.ac.uk.
}

DeChiara et al. 1991). By the mid-1990s, therefore, some of the cornerstones of imprinting research were in place. Recent significant additions to this puzzle are experiments showing how imprints are introduced in the parental germ cells by DNA methyltransferases and their cofactors (Bourc'his et al. 2001; Hata et al. 2002; Kaneda et al. 2004). How imprints are erased in early germ cells is still a mystery that needs to be explored.

Meanwhile, a large amount of information has been accumulated on individual imprinted genes (currently about 80 in mouse) and their functions in fetal growth control, neonatal physiology, and adult behavior (http://www. mgu.har.mrc.ac.uk/research/imprinting/). Once a number of imprinted genes had been isolated, it quickly became clear that most imprinted genes are clustered in the genome. Some of the larger clusters extend over 1-2 Mb (megabases) and contain several imprinted genes. The reason why imprinted genes are clustered in this way and the evolution of clusters are still not understood. However, it can be imagined that genetic conflict occurs at the level of gene products as well as at different mechanistic levels. For example, maternal expression of the H19 gene prevents maternal expression of the linked growth-enhancing Igf2 gene (see below). Paternal expression of the noncoding Air transcript prevents paternal expression of the overlapping growth suppressing $\operatorname{Ig} f 2 r$ gene. These mechanistic interactions ("battles") between imprinted genes are facilitated by physical linkage of the genes and shared regulatory elements.

Clustering and sharing of regulatory elements is indeed a common theme in imprinting. Such elements include enhancers, silencers, insulators, and activators, all of which have the potential to be epigenetically regulated. Of particular interest are the so called "imprinting centers" (ICs), which have the property that they regulate epigenetic modifications and imprinted expression of several genes throughout clusters. Their influence can extend over several hundred kilobases to megabases.

The purpose of this paper is to explore the nature of mechanistic interactions between imprinted genes in clusters. We describe recent insights into the Igf2-H19 region suggesting that reciprocal access to a single set of enhancers is regulated by chromatin "looping." In the ad- 
jacent imprinted domain, we argue that an imprinted noncoding transcript, Kcnq lot 1, is pivotal for the recruitment of repressive histone modifications leading to a silent chromatin structure in the adjacent genes. We further explore the general applicability of these two models of imprinting regulation to other imprinting clusters.

\section{THE IGF2-H19 LOCUS (IC1 DOMAIN)}

\section{Structure of the Locus and Regulatory Elements}

The fetal growth factor Insulin-like growth factor 2 gene $(\operatorname{Ig} f 2)$ is paternally expressed and lies upstream of the maternally expressed noncoding RNA gene $H 19$ (Fig. 1). The distance between $\operatorname{Ig} f 2$ and $H 19$ is $\sim 100 \mathrm{~kb}$. While the two genes are expressed from the opposite parental chromosomes, their spatial and temporal patterns of expression are strikingly similar. Both genes are expressed predominantly in mesodermal, endodermal, and extraembryonic tissues in the developing fetus, with a decline of expression during the first 3 weeks of postnatal life.

Lineage- and tissue-specific expression of both genes are governed by a number of different enhancers, most of which are located distal to H19 (Leighton et al. 1995; Kaffer et al. 2001; Davies et al. 2002). A differentially methylated region (DMR) with paternally derived germ line methylation is located $2 \mathrm{~kb}$ upstream of H19. This region acts as an insulator or boundary element when unmethylated; it has multiple binding sites for the insulator protein CTCF, which is bound to the maternal allele ( Bell and Felsenfeld 2000; Hark et al. 2000; Kanduri et al. 2000; Szabo et al. 2000; Arney 2003). CTCF binding is sensitive to DNA methylation, so the methylated paternal allele of the DMR does not bind CTCF. As a result, the insulator is inactive. The active insulator on the maternal allele is thought to restrict access of the enhancers exclusively to the $H 19$ promoter. On the paternal allele, by contrast, the $H 19$ promoter is methylated and so this gene is silent, but the insulator is now inactive, allowing the distal enhancers access to the Igf2 gene. Notably, the Igf2 promoters are not generally methylated on the inactive allele, and even show DNase hypersensitive sites, indicating that in principle the chromatin is transcriptionally permissive (Feil et al. 1995).

In addition to the distal enhancers and the insulator region, regulatory elements in $I g f 2$ are also important (Arney 2003). The Igf2 gene has three DMRs: DMR0 is maternally methylated and overlaps with the placental specific Igf2 promoter P0: DMR1 is paternally methylated and contains a methylation-sensitive silencer (Constancia et al. 2000; Eden et al. 2001); and the intragenic DMR2 is also paternally methylated and contains a methylation-sensitive activator (Murrell et al. 2001). These three DMRs are non-germ-line DMRs in that their differential methylation arises during early postimplantation development (Lopes et al. 2003). Importantly, deletion of the unmethylated DMR1 does lead to reactivation of the otherwise silent maternal Igf2 allele, in the presence of an apparently intact insulator (Constancia et al. 2000). Additional silencer and enhancer sequences are present in the locus; a more complete summary can be found in Arney (2003).

\section{Models for Igf2-H19 Imprinting}

An initial model for imprinting regulation in the locus was based on enhancer competition, whereby $H 19$ had privileged access to the enhancers, but when $H 19$ was methylated, Igf2 could gain access to them (Bartolomei

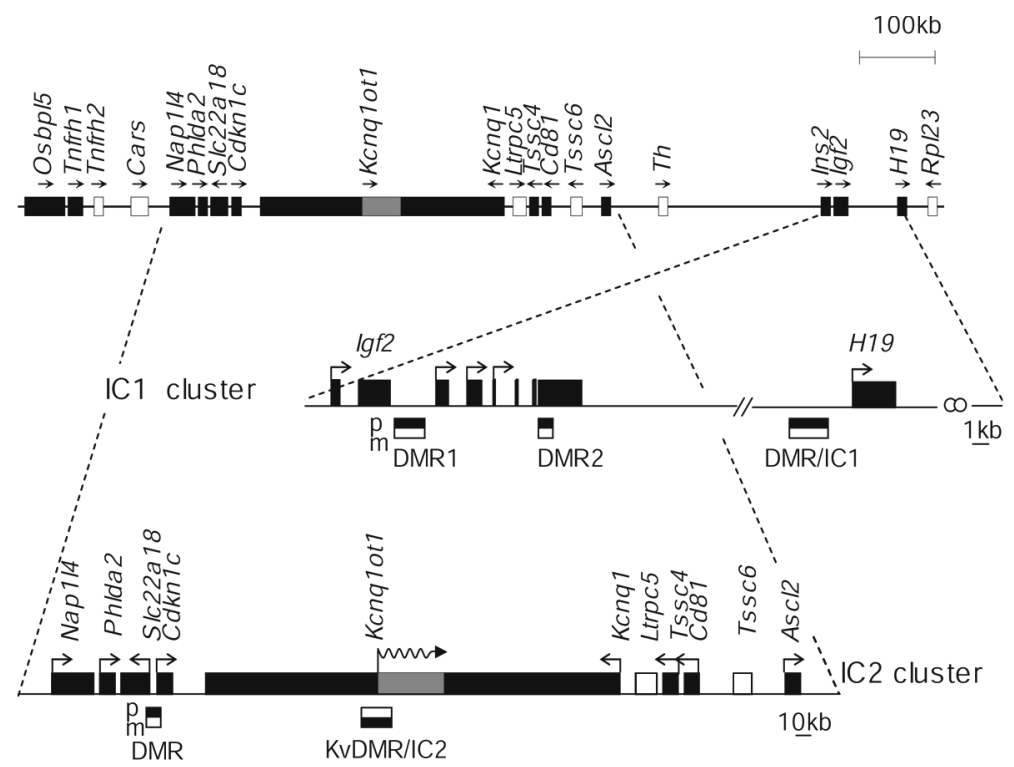

Figure 1. A map showing the two imprinting clusters on mouse distal chromosome 7. (Top) The relative positions of the IC1 and IC2 imprinting clusters and their respective ICs with imprinted genes indicated by filled boxes. (Middle) The IC1 cluster. The position of the DMRs is shown with a filled black box indicating the methylated allele and a white box for the unmethylated allele. The enhancers are represented by unfilled circles. (Bottom) The IC2 cluster with DMRs indicated as above. The Kcnqlot1 antisense transcript is represented by a curly arrow. 

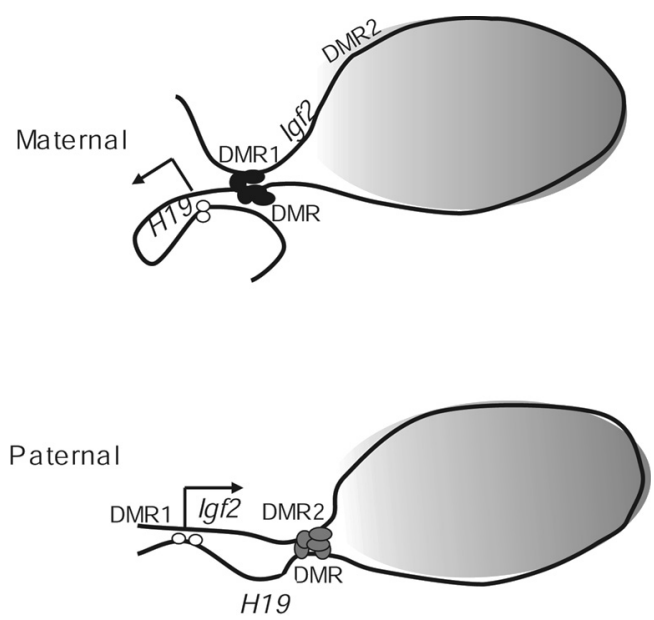

Figure 2. Parent-specific interactions between the DMRs provide an epigenetic switch for Igf2. On the maternal allele the unmethylated H19 DMR, which is bound by CTCF and possibly other proteins (stippled ovals), and Igf2 DMR1 interact, resulting in two chromatin domains, with the $H 19$ gene in an active domain with its enhancers (small circles) close to its promoter, and the $I g f 2$ gene in an inactive domain away from the enhancers (shaded area). On the paternal allele the methylated H19 DMR associates with the methylated Igf2 DMR2 through putative protein factors (filled ovals), moving Igf2 into the active chromatin domain. The location of DMR2 at the end of the Igf 2 gene positions its promoters in close vicinity to the enhancers downstream of H19. H19 remains in the active domain but is silenced by DNA methylation.

and Tilghman 1992). This model was superseded by the current one in which the H19 DMR is shown to have methylation-sensitive insulator function, which depends on CTCF binding. How do insulators work? They are defined as elements that block promoter enhancer communication (Labrador and Corces 2002); recently CTCF has been found to bind nucleophosmin, which itself appears to be tethered to the nucleolar surface (Yusufzai et al. 2004). Such an anchoring of insulators to nuclear substructures could lead to the formation of chromatin loops that separate promoters and enhancers so that they can no longer interact (Fig. 2). Interestingly, a chromatin loop model was proposed some time ago for Igf2 and H19, in which they come into close physical proximity, with different contact points on the maternal and paternal chromosomes (Fig. 2) (Banerjee and Smallwood 1995).

Another issue that needs to be considered in this context is the observation that epigenetic marks in clusters can be under the control of imprinting centers. Thus in Prader-Willi/Angelman syndrome, for example, mutations in the PWS IC on the paternal allele lead to methylation and silencing of multiple linked imprinted genes (Nicholls et al. 1998). In the mouse Igf2-H19 locus, maternal deletion of the H19 DMR (=IC1) leads to methylation of the maternal DMR1 and DMR2 in Igf2 (Forne et al. 1997; Lopes et al. 2003). How this coordination of epigenetic marks in a cluster works and whether it is linked to the sharing of regulatory regions between the genes are unknown. However, some interesting observations have recently been made. First, in the case of the H19 DMR, CTCF protein itself is apparently involved in protecting the maternal DMR (to which it binds) from de novo methylation during early postimplantation development (Schoenherr et al. 2003; Szabo et al. 2004). Second, while DMR1 and DMR2 in Igf2 are actually differentially methylated in the germ line (paternal methylation) this methylation is lost soon after fertilization, but is then regained postimplantation. Importantly, if the maternal H19 DMR is deleted, the maternal DMR1 and DMR2 become methylated at the same stage, suggesting that they are normally protected from de novo methylation by an intact H19 DMR (Lopes et al. 2003) (whether this also involves CTCF is not known). From these observations, we developed a model in which on the maternal allele the H19 DMR loops together with Igf2 DMR1 (and perhaps also DMR2). When the loop is disrupted by deletion of the H19 DMR, Igf2 DMRs 1 and 2 are no longer protected from de novo methylation (Fig. 2) (Lopes et al. 2003). This model is complementary to the Banerjee and Smallwood model, but they make different predictions about where physical contacts are to be expected.

\section{Testing the Loop Model}

Chromosome looping was proposed many years ago to underlie the action of distant enhancer elements on proximal promoters, especially in more complex vertebrate genomes (Bulger and Groudine 1999). Only recently, however, have technological developments allowed these models to be directly tested. The first method, chromosome conformation capture (3C), is based on cutting cross-linked chromatin with defined restriction enzymes, and religating the ends under very dilute DNA concentrations (Dekker et al. 2002). Under these conditions, restriction sites from remote genomic regions can ligate to each other if they were in close physical proximity when the chromatin was cross-linked. Thus, successful PCR amplification with primers from the two remote regions would indicate physical proximity of the remote regions. In the mammalian system, this technique has been applied to study the developmental regulation of beta globin gene expression (Tolhuis et al. 2002). A remote enhancer (the LCR) has indeed been shown to come into close physical contact with the gene with the intervening DNA looping out. These interactions are dynamic and are regulated in a tissue and developmental fashion (Palstra et al. 2003).

The second method has also been developed to look at beta globin expression and is based on cross-linking locally an RNA FISH probe to the nuclear RNA as it is transcribed (of the globin gene in fetal blood cells in this case), followed by pulldown of the crosslinked protein-nucleic acid complex using a tag on the FISH probe. The complex is then analyzed to see which remote sequences are trapped. As with the $3 \mathrm{C}$ technique, areas of the enhancer (LCR) were found to contact the gene in tissues in which transcription occurs (Carter et al. 2002). The confirmation of "looping" with different techniques is important, and inspires confidence in these new technologies.

We chose to test the looping model for Igf2-H19 by two different techniques. First, we reasoned that chromatin immunoprecipitation (ChIP) could be used with an antibody specific to a protein located on the H19 DMR in 
order to see whether areas in $I g f 2$ would be coprecipitated. However, a protein such as CTCF (which is bound to the maternal H19 DMR) is so ubiquitous that it cannot be used for this purpose. We thus decided to engineer a H19 DMR with a unique protein tag. Three binding sequences for the yeast Gal4 transcriptional activator protein (termed UAS) were inserted into the H19 DMR by homologous recombination in ES cells and knockin mice were generated (Murrell et al. 2004). These mice were then bred with transgenic mice ubiquitously expressing the DNA-binding domain of the Gal4 protein fused to a unique peptide tag (human MYC). Following ChIP with a MYC tag antibody, the DMRs and other regions were analyzed by Q-PCR. The H19 DMR showed strong enrichment with the antibody, confirming that the knockin strategy was working and that the Gal4-MYC protein was indeed located at the H19 DMR with the inserted UAS sequences. Interestingly, with maternal transmission of the modified DMR, DMR 1 of Igf 2 was also enriched, but neither DMR2 nor intervening sequences between $\operatorname{Ig} f 2$ and $H 19$ were. With paternal transmission, by contrast, neither DMR1 nor intervening sequences were enriched, but DMR2 was. The preliminary conclusions from this experimental system were that on the maternal chromosome, the $H 19$ DMR was closely associated with Igf2 DMR1, whereas on the paternal chromosome it was associated with DMR2.

We applied the $3 \mathrm{C}$ method in order to confirm these results. Ligation products were indeed detected between the H19 DMR and DMR2, and the H19 DMR and DMR1. For DMR2, it was possible to determine by sequence polymorphisms that it was the paternal allele that was associated with the H19 DMR. Thus the combined evidence from the different techniques suggests that the H19 DMR comes in close proximity to DMR1 on the maternal allele and to DMR2 on the paternal one. It should be noted that these analyses are not quantitative, and so the proportion of cells in any one tissue that contain the loop structures remains to be determined. The loops we see may be only transient and may or may not be present in nonexpressing cells. It will also be of interest to see whether other sequences between $\operatorname{Ig} f 2$ and H19, or those further away from the genes, particularly Ins2, which is also paternally expressed in some tissues, come into physical proximity with the DMRs.

\section{An Epigenetic Switch}

The model arising from these observations is simple (Fig. 2). On the maternal allele the unmethylated H19 DMR and Igf2 DMR1 come together; proteins involved in these interactions may well include CTCF and others. This places Igf2 inside a loop and insulates it from the enhancers. On the paternal allele, Igf2 moves out of the loop since the H19 DMR and DMR2 are now interacting; this allows interaction between the enhancers and $I g f 2$. While H19 remains outside of the "silent" loop, it is inactivated by DNA methylation. CTCF cannot be involved in the interactions between the H19 DMR and DMR2 because it does not bind to the methylated alleles. Whether proteins that bind preferentially to methylated DNA such as MBDs are involved in these interactions needs to be established. In human fibroblast cells the $3 \mathrm{C}$ method revealed further allele-specific interactions between restriction fragments containing the promoters of IGF2 and H19 and a fragment carrying their (presumptive) shared enhancers (Fig. 2) (Y.Yang and M. Higgins, unpubl.). Presumably these enhancer/promoter interactions are mediated by additional higher-order chromatin structures, perhaps involving a putative CTCF-dependent insulator (conserved between mouse and humans) that lies downstream of the enhancers (Ishihara and Sasaki 2002). Potential involvement of CTCF in looping can now be tested genetically using mutant DMRs that lack CTCF-binding sites (Pant et al. 2003; Schoenherr et al. 2003; Szabo et al. 2004), and whether methylation of the paternal DMR is needed for looping can be tested using a mutant DMR that lacks CpGs (M. Bartolomei, pers. comm.). These experiments may begin to reveal the cis- and trans-acting requirements for looping (Patrinos et al. 2004). It should be noted that our model is consistent with most knockout studies of the DMRs (Arney 2003). Knockouts of H19 DMR or the Igf2 DMRs may disrupt the looping structures. Upon maternal transmission of a H19 DMR deletion, reduced H19 expression accompanied by activated $I g f 2$ expression was observed, while upon paternal transmission of the deletion, reduced Igf 2 expression accompanied by activated H19 expression was observed (Thorvaldsen et al. 1998). (It is noteworthy that the expression levels of the activated genes were still significantly lower than the wild type.) Thus, without the $H 19$ DMR, both the Igf2 and the H19 genes are not restricted to a particular silent or active domain. Deletion of the DMR1 of $\operatorname{Igf} 2$ leads to biallelic $\operatorname{Ig} f 2$ expression upon maternal transmission (Constancia et al. 2000), while deletion of the DMR2 of Igf2 leads to transcriptional down-regulation of Igf 2 upon paternal transmission (Murrell et al. 2001), which is consistent with DMR1 and DMR2 contributing to the looping structure on the maternal and paternal alleles, respectively.

A recent study shows that the paternal DMR2 is associated with the nuclear matrix in Igf2-expressing cells, so this may provide an anchor point for the base of the loop (Weber et al. 2003). The same has recently been observed for an insulator element upstream of the chicken beta globin genes (Yusufzai and Felsenfeld 2004), and CTCF has been found to bind to nucleophosmin, which can tether it to the nucleolar membrane (Yusufzai et al. 2004). Such interactions with subnuclear structures may well underlie the formation or maintenance of functional chromatin loops. In this way our observations would add to the growing body of evidence suggesting that regulatory elements and gene promoters in vertebrates are kept under tight control by higher-order chromatin structures, especially loops (Chubb and Bickmore 2003). The fascinating aspect of the observations on $\operatorname{Igf2-H19}$ is that these higher-order structures can be epigenetically regulated, thus providing simple epigenetic switches. It will be interesting to see whether other imprinted loci, or other epigenetically regulated loci, such as random monoallelically expressed genes (Chess 1998), possess similar epigenetic switches. 


\section{THE Kcnq1ot1 LOCUS (IC2 DOMAIN)}

\section{Structure of the Locus and Regulatory Elements}

The IC2 domain is located further telomeric from the IC1 domain but the two imprinted domains are closely linked (Fig. 1). Nevertheless, most of the genetic evidence available indicates that imprinting in the two domains is independently regulated. The IC2 domain contains the antisense RNA gene Kcnq1ot1, whose promoter region is currently defined as IC2. The Kcnq1ot1 gene is located within an intron of the Kcnql gene and its promoter overlaps with a DMR with germ line methylation arising from the oocyte (Engemann et al. 2000). The unmethylated paternal allele of Kcnq1ot 1 is therefore transcribed while the maternal allele remains transcriptionally silent. All other genes in the $\sim 800-\mathrm{kb}$ domain surrounding IC2 are maternally expressed (or not imprinted; Fig. 1). The Kcnqlot1 transcript partially overlaps with the large Kcnql gene, but this overlap probably does not extend to the Kcnql promoter region. A number of the genes in the IC2 domain are imprinted only in the placenta, attesting perhaps to the role in placental growth and function of a number of genes in the IC1 and IC2 domains. What is surprising is that most imprinted genes in the IC2 region do not have DMRs; this seems to be quite different from other larger imprinting clusters such as the PWS/AS region (Nicholls et al. 1998; A. Lewis et al., unpubl.).

The functional definition of the IC2 domain comes from knockout studies of the Kcnqlotl promoter region, both in cell lines and in mice. These show that if this region is removed from the paternal chromosome, the otherwise silent genes flanking it are now expressed (Horike et al. 2000; Fitzpatrick et al. 2002). This has been tested as far as Ascl2 on the centromeric side and Phlda2 on the telomeric side. By this criterion, the imprinted domain regulated by IC2 is at least $800 \mathrm{~kb}$ in size. Ascl2 is very likely the most centromeric imprinted gene in the domain, but the telomeric end has not been defined. It is unclear from these studies if it is the DNA sequence at the Kcnqlot1 promoter (that was deleted in the IC2 knockout), the Kcnq1ot1 RNA, or the fact that the region is transcribed on the paternal chromosome that is responsible for cis-acting silencing. By transfection assays, the DNA segment itself appears to have methylation-sensitive silencer or insulator activity, depending on which cell line is used for the tests (Kanduri et al. 2002; Thakur et al. 2003). By analogy with the $\operatorname{Ig} f 2 r$ antisense gene Air, which is also paternally expressed, it is possible that the Kcnq1ot1 RNA (or the act of transcription) plays a role in silencing (Sleutels et al. 2002). This possibility is further strengthened by parallels with imprinted X-chromosome inactivation (below). What cis-acting sequences are responsible for imprinting and differential methylation of IC2 in the first place is unclear. A series of BAC and YAC transgenes containing IC2 and flanking sequences show that imprinting and the full tissue-specific pattern of expression (of $C d k n 1 c$ ) depend upon the presence of remote sequence elements (John et al. 2001; F. Cerrato et al., unpubl.).

\section{DNA Methylation Is Not Required for Maintenance of Imprinting: A Histone Methylation Imprint}

Because of the relative scarcity of DMRs we were curious to see if DNA methylation was involved in regulating imprinting in the IC2 region, especially in the placenta. We used the Dnmtlc mutation, which removes the catalytic domain of Dnmt1 and results in a nonfunctional enzyme (Lei et al. 1996). Genomic methylation is dramatically reduced. Indeed, the maternal methylation of the Kcnq1ot1 promoter was completely lost in both Dnmt1-/- embryos and Dnmt1-/- trophoblast (the precursor tissue of the placenta). This loss of DNA methylation causes a complete loss of imprinting at the IC2 cluster in the embryo. However, imprinted genes in the placenta that do not possess DMRs (such as Ascl2, Obph1, Kcnq1, etc.) do not lose imprinting (i.e., the paternal allele remains repressed) (A. Lewis et al., unpubl.). By contrast, the two genes with DMRs (Kcnq1ot1 and $C d k n 1 c)$ do lose imprinting and are now biallelically expressed. Thus the maintenance of placental imprinting in this region does not apparently require DNA methylation. We therefore asked if the imprinted genes were marked by other types of epigenetic modifications. Chromatin immunoprecipitation was carried out in an allele-specific way using antibodies against acetyl and methyl modifications of histone tails. The Montpellier group (D. Umlauf and R. Feil, pers. comm.) thus found that in the placenta an 800-kb region (at least) surrounding Kcnqlotl, and extending at least to Ascl2 on the centromeric and Obph1 on the telomeric side, was dimethylated at $\mathrm{H} 3 \mathrm{~K} 9$, and trimethylated at H3K27 (Fig. 3) (D. Umlauf et al., unpubl.). This is true not only of gene promoters, but also of all intra- and intergenic regions analyzed. By contrast, the maternal region in the placenta is marked with activating histone modifications (namely, H3K4 dimethylation and H3K9 and K14 acetylation). The only exception is the Kcnq1ot1 DMR itself, in which histone methylation at K9 and K27 correlates with DNA methylation, and therefore marks the maternal chromosome, both in the placenta and in the embryo. In the embryo such allele-specific histone modifications are found only at confined regions comprising the DMRs. Other studies by D. Umlauf and coworkers (unpubl.) show that this regional histone methylation imprint is already present in ES cells, and thus may arise very early in development. It is then maintained in extraembryonic tissues, but apparently lost in embryonic ones.

\section{The Repressive Histone Methylation Imprint Depends on IC2}

We next asked if the histone methylation imprint depended on the IC2. Indeed, with paternal transmission of the IC2 deletion, histone modification differences as judged by allele-specific ChIP were eliminated at the several genes we analyzed. This indicates that the repressive histone methylation on the paternal chromosome is not established or is not maintained in mice carrying an IC2 deletion (A. Lewis et al., unpubl.). As stated before, it is 

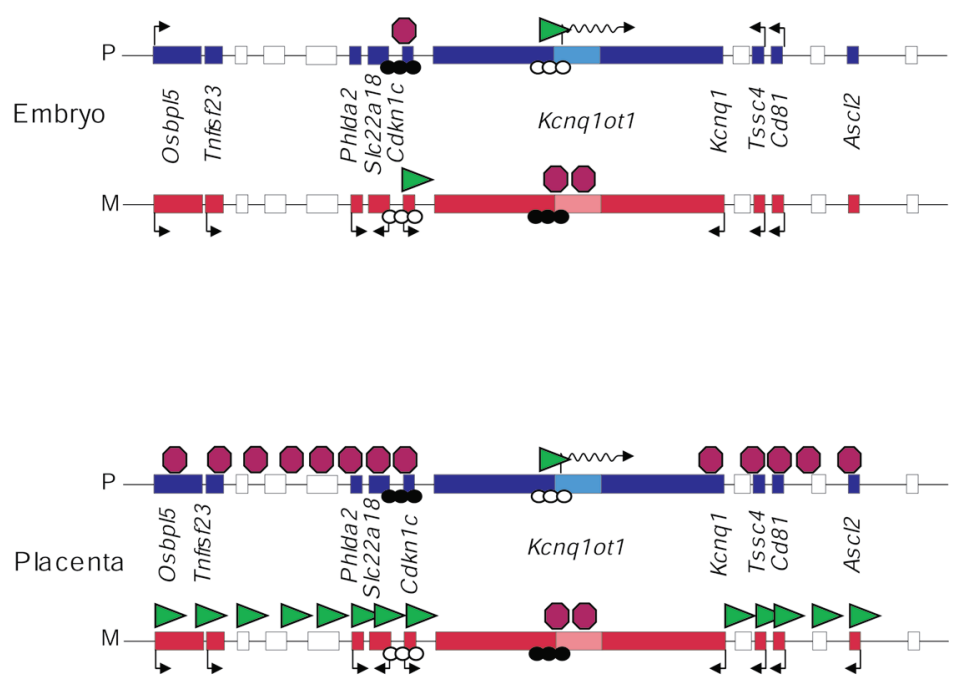

Figure 3. A summary of the differences in imprinted expression and histone modifications at the IC 2 cluster and between the embryo and placenta. Imprinted genes are colored blue on the paternal chromosome and red on the maternal chromosome. Nonimprinted genes are white. Arrows show expression status in the embryo or placenta. DMRs are represented by black circles on the methylated allele and white circles on the unmethylated allele. Green triangles represent "active" histone modifications such as H3 acetylation and H3 K4 dimethylation. Dark red hexagons represent "repressive" histone modifications such as H3 K9 dimethylation and H3 K27 trimethylation. Differential histone modification in the embryo is limited to the DMRs possibly because DNA methylation is somehow required for its maintenance in the embryo (Fournier et al. 2002). In the placenta, however, the entire imprinted domain is marked by repressive modifications on the paternal allele and active modifications on the maternal allele.

not yet clear whether the DNA element at IC2, Kcnq1ot1 transcription, or the Kcnqlot 1 transcript is responsible for attracting repressive histone modifications to the region in cis. Our favored model is that the transcript itself plays some role. Thus we envisage (Fig. 4) that the Kcnqlot1

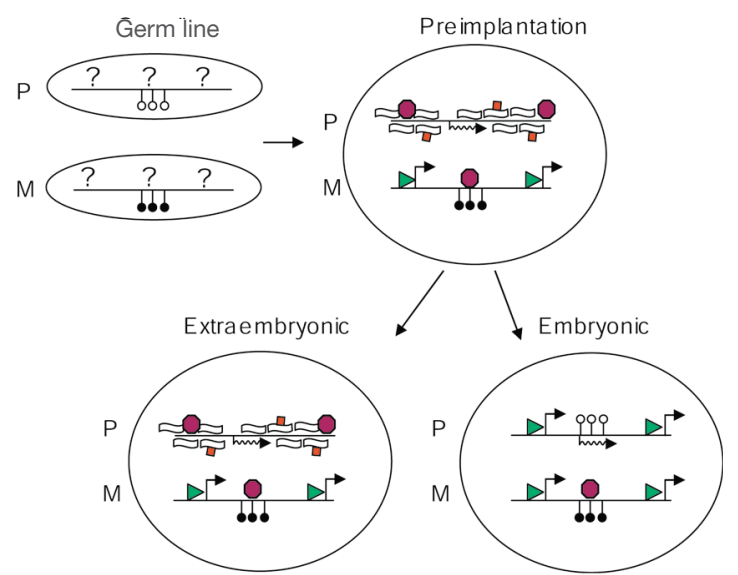

Figure 4. Model of the establishment of imprinting at the IC2 locus. Arrows show active gene transcription. Transcription of the antisense transcript is shown by a curly arrow. DMRs are represented by black circles on the methylated allele and white circles on the unmethylated allele. Green triangles represent histone acetylation or $\mathrm{H} 3 \mathrm{~K} 4$ dimethylation and red hexagons represent histone $\mathrm{K} 9$ or $\mathrm{K} 27$ methylation. RNA coating the chromosome is shown by a wavy rectangle. Histone methyltransferases are shown as red boxes. The germ line histone modification status is as yet unknown. In preimplantation embryos we predict that Kcnqlot 1 is expressed from the paternal allele and coats the chromosome. This coating may recruit histone modifications possibly using a mechanism similar to Xist RNA in X-inactivation. These modifications are then maintained in the extraembryonic lineages but lost in embryonic lineages.
RNA is paternally expressed early in the preimplantation embryo. We then envisage that the noncoding Kcnq1ot 1 RNA coats the IC2 region, and that this recruits, directly or indirectly, histone methyltransferases to the paternal chromosome. Indeed Umlauf et al. (unpubl.) show that the Eed/Ezh2 polycomb complex is recruited to the paternal chromosome in ES cells. This complex has K27 and some K9 methyltransferase activity. Other methyltransferases, such as perhaps G9a, might be recruited to methylate the K9 residue. Indeed, it is interesting that in the Eed knockout mouse some of the paternally silenced genes analyzed here are derepressed, but not all of them are (Mager et al. 2003). Therefore differential, and perhaps also additive, effects of the different repressive histone modifications might be imagined. In addition, it is likely that histone methylation and DNA methylation cooperate in silencing. For example, the silent copies of Kcnqlot1 and of Cdkn1c (which possess DMRs) are considerably "more silent" than those of Kcnq1 or Cd81 (no DMRs), which show a bias only in parental expression.

We have shown that the histone methylation imprint, once established, is independent of DNA methylation. But if our model is correct the IC2 domain may require the continued presence of the Kcnqlot1 RNA to maintain imprinting. So in the Dnmt1-/- placenta, why does loss of methylation from the maternal Kcnq1ot1 promoter (causing derepression of maternal Kcnq1ot1 transcription) not result in silencing of the maternal chromosome? In heterozygous crosses between Dnmt1-/wt animals, the maternal oocyte still contains large amounts of Dnmt1 protein, which may become depleted only toward the blastocyst stage (at which stage the histone methylation imprint is already established; D. Umlauf et al., unpubl.). Thus activation of the maternal copy of Kcnq1ot1 may 
occur only around the blastocyst stage at which perhaps a "window of opportunity" for cis establishment of the histone methylation imprint is already closed.

Why is histone methylation apparently important in the placenta, but less so in the embryo, for imprinting? It is possible that imprinting coevolved with the placenta in mammalian radiation, and that initially imprinted genes acted primarily in the placenta to regulate supply of maternal nutrients to the fetus (Reik et al. 2003). We speculate that during this phase of imprinting evolution, histone methylation may have been the primary epigenetic mark involved in imprinting. In marsupials, for example, the Insulin-like growth factor 2 receptor gene is imprinted as it is in mice, but the DMR2 region, which in mice carries the DNA methylation imprint, is not methylated (Killian et al. 2000). Similarly, inactivated genes on the X chromosome are not methylated in female marsupials, but they are in female mice (Wakefield et al. 1997). Thus an ancestral imprinting mechanism may have been based on histone modifications and was perhaps initially limited to the placenta. Since we believe a mechanism based on only histone modifications to be relatively "unstable" or "leaky," later evolution of imprinted gene expression in fetal tissues and after birth made necessary the recruitment of a more stable epigenetic mark, namely DNA methylation.

\section{The Insulator-Loop Model and the Noncoding RNA Model: Application to Other Imprinted Regions}

The noncoding RNA model is clearly applicable to other imprinted regions as well. First, there are overt similarities with imprinted X-chromosome inactivation. The current model of imprinted Xi (in the mouse placenta) envisages that Xist is expressed from the paternal $\mathrm{X}$ as soon as the two-cell stage, and begins to coat the inactivating $X$ soon after. Histone methylation recruitment also begins during preimplantation development, and so does gene inactivation along the paternal $\mathrm{X}$ chromosome, possibly in a gradient from the Xist locus (Huynh and Lee 2003; Mak et al. 2004; Okamoto et al. 2004). What is still debated is whether, in addition to early postzygotic action of Xist, the paternal $\mathrm{X}$ is already marked or preinactivated in the germ line, perhaps because of the X-inactivation event that occurs during spermatogenesis (Huynh and Lee 2003). Notwithstanding this uncertainty, it is generally thought that in the morula and early blastocyst, the majority of cells, including those in the inner cell mass, carry an inactive paternal $\mathrm{X}$ chromosome, and that this silencing is erased (starting with down-regulation of Xist) in the ICM, followed by random $\mathrm{X}$-inactivation at a slightly later stage in the epiblast (Mak et al. 2004; Okamoto et al. 2004).

We think that the noncoding RNA model is potentially applicable to a number of other imprinted loci, particularly those with paternal expression of noncoding antisense transcripts. The $\operatorname{Ig} f 2 r$ locus is especially interesting since the $\operatorname{Igf} 2 r$ gene is overlapped by the noncoding antisense transcript Air, and the Air transcript or transcription is needed for $c i$ inactivation of $\operatorname{Ig} f 2 r$ as well as the two linked genes, Slc22a2 and Slc22a3, which are only imprinted in the placenta (and do not have DMRs; Sleutels et al. 2002). Thus our specific prediction for this locus would be that in the placenta there should be a histone methylation imprint in Slc22a2 and Slc22a3, and that genetic removal of DNA methylation should not lead to loss of imprinting of these Slc22a genes. The same model may well be applicable to other clusters with maternal germ line methylation and a role in placental growth and function. For example, the Snurf-Snrpn promoter transcribes a very long paternal transcript which may have a role in cis-inactivation.

Conversely, we speculate that the looping-insulator model may be more widely applicable to clusters with paternal germ line methylation, such as Dlk1-Gtl2, and Rasgrf1. Maternal deletion of the (unmethylated) DMR upstream of Gtl2 leads to reactivation of the normally silent Dlkl on the maternal chromosome, suggesting disruption of an insulator similar to that seen upstream of H19 (Lin et al. 2003). Rasgrfl has a paternally methylated DMR with insulator activity (Yoon et al. 2002).

We must also consider the possibility that these two models are not necessarily mutually exclusive. Much of the regulatory machinery important for the epigenetic switch in the Igf2-H19 region is also present in the IC2 cluster. For instance the Kcnq1ot1 DMR has been shown to have methylation-sensitive insulator function in several somatic cell lines (Kanduri et al. 2002). Interestingly, in trophoblast-derived cells the DMR functions as a bidirectional silencer rather than a unidirectional insulator (Thakur et al. 2003). Perhaps these tissue-specific effects contribute to the differences in imprinted expression in embryonic and extraembryonic lineages. Perhaps the DMR region is also involved in a tissue-specific higherorder chromatin structure that isolates promoters and enhancers, or that isolates the IC2 cluster (and its allele-specific histone modifications) from the surrounding loci. The Igf2-H19 cluster contains several noncoding transcripts including $H 19$ itself. None appear to be as extensive as the Kcnqlotl transcript, which is at least $60 \mathrm{~kb}$ long, so a coating mechanism as described above seems unlikely. However, the process of transcription of these noncoding RNAs or the RNAs themselves may contribute to transcriptional, posttranscriptional, or RNAilike mechanisms of gene regulation.

\section{ACKNOWLEDGMENTS}

We are grateful to many colleagues who have contributed to this work through discussions of ideas and comments on the manuscript, particularly P. Smith, J. Walter, G. Kelsey, P. Fraser, M. Constancia, F. Santos, A. Ferguson-Smith, and A. Riccio. The authors' work is funded by BBSRC, MRC, and CRUK. R.F. acknowledges grant funding from ARC. D.U. is a recipient of a Ministry of Research fellowship.

\section{REFERENCES}

Arney K.L. 2003. H19 and Igf2-Enhancing the confusion? Trends Genet. 19: 17.

Banerjee S. and Smallwood A. 1995. A chromatin model of IGF2/H19 imprinting. Nat. Genet. 11: 237.

Barlow D.P., Stoger R., Herrmann B.G., Saito K., and Schweifer 
N. 1991. The mouse insulin-like growth factor type-2 receptor is imprinted and closely linked to the Tme locus. Nature 349: 84.

Bartolomei M.S. and Tilghman S.M. 1992. Parental imprinting of mouse chromosome 7. Semin. Dev. Biol. 3: 107.

Bartolomei M.S., Zemel S., and Tilghman S.M. 1991. Parental imprinting of the mouse H19 gene. Nature 351: 153.

Bell A.C. and Felsenfeld G. 2000. Methylation of a CTCF-dependent boundary controls imprinted expression of the Igf2 gene. Nature 405: 482.

Bourc'his D., Xu G.L., Lin C.S., Bollman B., and Bestor T.H. 2001. Dnmt3L and the establishment of maternal genomic imprints. Science 294: 2536.

Brandeis M., Kafri T., Ariel M., Chaillet J.R., McCarrey J., Razin A., and Cedar H. 1993. The ontogeny of allele-specific methylation associated with imprinted genes in the mouse. EMBO J. 12: 3669.

Bulger M. and Groudine M. 1999. Looping versus linking: Toward a model for long-distance gene activation. Genes Dev. 13: 2465

Carter D., Chakalova L., Osborne C.S., Dai Y.F., and Fraser P. 2002. Long-range chromatin regulatory interactions in vivo. Nat. Genet. 32: 623.

Chess A. 1998. Expansion of the allelic exclusion principle? Science 279: 2067.

Chubb J.R. and Bickmore W.A. 2003. Considering nuclear compartmentalization in the light of nuclear dynamics. Cell 112: 403.

Constancia M., Dean W., Lopes S., Moore T., Kelsey G., and Reik W. 2000. Deletion of a silencer element in Igf2 results in loss of imprinting independent of H19. Nat. Genet. 26: 203.

Davies K., Bowden L., Smith P., Dean W., Hill D., Furuumi H., Sasaki H., Cattanach B., and Reik W. 2002. Disruption of mesodermal enhancers for Igf 2 in the minute mutant. Development 129: 1657.

DeChiara T.M., Robertson E.J., and Efstratiadis A. 1991. Parental imprinting of the mouse insulin-like growth factor II gene. Cell 64: 849 .

Dekker J., Rippe K., Dekker M., and Kleckner N. 2002. Capturing chromosome conformation. Science 295: 1306.

Delaval K. and Feil R. 2004. Epigenetic regulation of mammalian genomic imprinting. Curr. Opin. Genet. Dev. 14: 188.

Eden S., Constancia M., Hashimshony T., Dean W., Goldstein B., Johnson A.C., Keshet I., Reik W., and Cedar H. 2001. An upstream repressor element plays a role in Igf2 imprinting. EMBO J. 20: 3518.

Engemann S., Strodicke M., Paulsen M., Franck O., Reinhardt R., Lane N., Reik W., and Walter J. 2000. Sequence and functional comparison in the Beckwith-Wiedemann region: Implications for a novel imprinting centre and extended imprinting. Hum. Mol. Genet. 9: 2691.

Feil R., Handel M.A., Allen N.D., and Reik W. 1995. Chromatin structure and imprinting: Developmental control of DNase-I sensitivity in the mouse insulin-like growth factor 2 gene. Dev. Genet. 17: 240.

Ferguson-Smith A.C. and Surani M.A. 2001. Imprinting and the epigenetic asymmetry between parental genomes. Science 293: 1086.

Fitzpatrick G.V., Soloway P.D., and Higgins M.J. 2002. Regional loss of imprinting and growth deficiency in mice with a targeted deletion of KVDMR1. Nat. Genet. 32: 426.

Forne T., Oswald J., Dean W., Saam J.R., Bailleul B., Dandolo L., Tilghman S.M., Walter J., and Reik W. 1997. Loss of the maternal $\mathrm{H} 19$ gene induces changes in Igf2 methylation in both cis and trans. Proc. Natl. Acad. Sci. 94: 10243.

Fournier C., Goto Y., Ballestar E., Delaval K., Hever A.M., Esteller M., and Feil R. 2002. Allele-specific histone lysine methylation marks regulatory regions at imprinted mouse genes. $E M B O J .21: 6560$.

Haig D. and Westoby M. 1989. Parent-specific gene expression and the triploid endosperm. Am. Nat. 134: 147.

Hark A.T., Schoenherr C.J., Katz D.J., Ingram R.S., Levorse J.M., and Tilghman S.M. 2000. CTCF mediates methylationsensitive enhancer-blocking activity at the H19/Igf2 locus.
Nature 405: 486.

Hata K., Okano M., Lei H., and Li E. 2002. Dnmt3L cooperates with the Dnmt3 family of de novo DNA methyltransferases to establish maternal imprints in mice. Development 129: 1983.

Horike S., Mitsuya K., Meguro M., Kotobuki N., Kashiwagi A., Notsu T., Schulz T.C., Shirayoshi Y., and Oshimura M. 2000. Targeted disruption of the human LIT1 locus defines a putative imprinting control element playing an essential role in Beckwith-Wiedemann syndrome. Hum. Mol. Genet. 9: 2075.

Huynh K.D. and Lee J.T. 2003. Inheritance of a pre-inactivated paternal X chromosome in early mouse embryos. Nature $\mathbf{4 2 6}$ : 857.

Ishihara K. and Sasaki H. 2002. An evolutionarily conserved putative insulator element near the $3^{\prime}$ boundary of the imprinted Igf2/H19 domain. Hum. Mol. Genet. 11: 1627.

John R.M., Ainscough J.F., Barton S.C., and Surani M.A. 2001. Distant cis-elements regulate imprinted expression of the mouse p57( Kip2) (Cdkn1c) gene: Implications for the human disorder, Beckwith-Wiedemann syndrome. Hum. Mol. Genet. 10: 1601

Kaffer C.R., Grinberg A., and Pfeifer K. 2001. Regulatory mechanisms at the mouse Igf2/H19 locus. Mol. Cell. Biol. 21: 8189.

Kanduri C., Fitzpatrick G., Mukhopadhyay R., Kanduri M., Lobanenkov V., Higgins M., and Ohlsson R. 2002. A differentially methylated imprinting control region within the Kcnq1 locus harbors a methylation-sensitive chromatin insulator. J. Biol. Chem. 277: 18106.

Kanduri C., Pant V., Loukinov D., Pugacheva E., Qi C.F., Wolffe A., Ohlsson R., and Lobanenkov V.V. 2000. Functional association of CTCF with the insulator upstream of the $\mathrm{H} 19$ gene is parent of origin-specific and methylation-sensitive. Curr. Biol. 10: 853.

Kaneda M., Okano M., Hata K., Sado T., Tsujimoto N., Li E., and Sasaki H. 2004. Essential role for de novo DNA methyltransferase Dnmt3a in paternal and maternal imprinting. $\mathrm{Na}$ ture 429: 900.

Killian J.K., Byrd J.C., Jirtle J.V., Munday B.L., Stoskopf M.K., MacDonald R.G., and Jirtle R.L. 2000. M6P/IGF2R imprinting evolution in mammals. Mol. Cell 5: 707.

Labrador M. and Corces V.G. 2002. Setting the boundaries of chromatin domains and nuclear organization. Cell 111: 151.

Lei H., Oh S.P., Okano M., Juttermann R., Goss K.A., Jaenisch R., and Li E. 1996. De novo DNA cytosine methyltransferase activities in mouse embryonic stem cells. Development 122: 3195.

Leighton P.A., Saam J.R., Ingram R.S., Stewart C.L., and Tilghman S.M. 1995. An enhancer deletion affects both H19 and Igf2 expression. Genes Dev. 9: 2079.

Lin S.P., Youngson N., Takada S., Seitz H., Reik W., Paulsen M., Cavaille J., and Ferguson-Smith A.C. 2003. Asymmetric regulation of imprinting on the maternal and paternal chromosomes at the Dlk1-Gt12 imprinted cluster on mouse chromosome 12. Nat. Genet. 35: 97.

Lopes S., Lewis A., Hajkova P., Dean W., Oswald J., Forne T., Murrell A., Constancia M., Bartolomei M., Walter J., and Reik W. 2003. Epigenetic modification in an imprinting cluster are controlled by a hierarchy of DMRs suggesting longrange chromatin interactions. Hum. Mol. Genet. 12: 295.

Mager J., Montgomery N.D., de Villena F.P., and Magnuson T. 2003. Genome imprinting regulated by the mouse Polycomb group protein Eed. Nat. Genet. 33: 502.

Mak W., Nesterova T.B., de Napoles M., Appanah R., Yamanaka S., Otte A.P., and Brockdorff N. 2004. Reactivation of the paternal X chromosome in early mouse embryos. Science 303: 666.

McGrath J. and Solter D. 1984. Completion of mouse embryogenesis requires both the maternal and paternal genomes. Cell 37: 179 .

Murrell A., Heeson S., and Reik W. 2004. Physical contact between differentially methylated regions partitions the imprinted $I g f 2$ and $H 19$ genes into parent specific chromatin loops. Nat. Genet. 36: 889.

Murrell A., Heeson S., Bowden L., Constancia M., Dean W., 
Kelsey G., and Reik W. 2001. An intragenic methylated region in the imprinted Igf2 gene augments transcription. EMBO Rep. 2: 1101.

Nicholls R.D., Saitoh S., and Horsthemke B. 1998. Imprinting in Prader-Willi and Angelman syndromes. Trends Genet. 14: 194.

Okamoto I., Otte A.P., Allis C.D., Reinberg D., and Heard E. 2004. Epigenetic dynamics of imprinted X inactivation during early mouse development. Science 303: 644.

Palstra R.J., Tolhuis B., Splinter E., Nijmeijer R., Grosveld F., and de Laat W. 2003. The beta-globin nuclear compartment in development and erythroid differentiation. Nat. Genet. 35: 190.

Pant V., Mariano P., Kanduri C., Mattsson A., Lobanenkov V., Heuchel R., and Ohlsson R. 2003. The nucleotides responsible for the direct physical contact between the chromatin insulator protein CTCF and the H19 imprinting control region manifest parent of origin-specific long-distance insulation and methylation-free domains. Genes Dev. 17: 586.

Patrinos G.P., de Krom M., de Boer E., Langeveld A., Imam A.M., Strouboulis J., de Laat W., and Grosveld F.G. 2004. Multiple interactions between regulatory regions are required to stabilize an active chromatin hub. Genes Dev. 18: 1495.

Reik W. and Walter J. 2001. Genomic imprinting: Parental influence on the genome. Nat. Rev. Genet. 2: 21.

Reik W., Constancia M., Fowden A., Anderson N., Dean W., Ferguson-Smith A., Tycko B., and Sibley C. 2003. Regulation of supply and demand for maternal nutrients in mammals by imprinted genes. J. Physiol. 547: 35.

Sasaki H., Jones P.A., Chaillet J.R., Ferguson-Smith A.C., Barton S.C., Reik W., and Surani M.A. 1992. Parental imprinting: Potentially active chromatin of the repressed maternal allele of the mouse insulin-like growth factor II (Igf2) gene. Genes Dev. 6: 1843.

Schoenherr C.J., Levorse J.M., and Tilghman S.M. 2003. CTCF maintains differential methylation at the Igf2/H19 locus. Nat. Genet. 33: 66.

Sleutels F. and Barlow D.P. 2002. The origins of genomic imprinting in mammals. Adv. Genet. 46: 119.

Sleutels F., Zwart R., and Barlow D.P. 2002. The non-coding Air RNA is required for silencing autosomal imprinted genes. $\mathrm{Na}$ ture 415: 810 .

Stoger R., Kubicka P., Liu C.G., Kafri T., Razin A., Cedar H., and Barlow D.P. 1993. Maternal-specific methylation of the imprinted mouse Igf2r locus identifies the expressed locus as carrying the imprinting signal. Cell 73: 61 .

Surani M.A., Barton S.C., and Norris M.L. 1984. Development of reconstituted mouse eggs suggests imprinting of the genome during gametogenesis. Nature 308: 548 .

Szabo P., Tang S.H., Rentsendorj A., Pfeifer G.P., and Mann J.R. 2000. Maternal-specific footprints at putative CTCF sites in the H19 imprinting control region give evidence for insulator function. Curr. Biol. 10: 607.

Szabo P.E., Tang S.H., Silva F.J., Tsark W.M., and Mann J.R. 2004. Role of CTCF binding sites in the Igf2/H19 imprinting control region. Mol. Cell. Biol. 24: 4791.

Thakur N., Kanduri M., Holmgren C., Mukhopadhyay R., and Kanduri C. 2003. Bidirectional silencing and DNA methylation-sensitive methylation-spreading properties of the Kcnq1 imprinting control region map to the same regions. J. Biol. Chem. 278: 9514.

Thorvaldsen J.L., Duran K.L., and Bartolomei M.S. 1998. Deletion of the H19 differentially methylated domain results in loss of imprinted expression of H19 and Igf2. Genes Dev. 12: 3693.

Tolhuis B., Palstra R.J., Splinter E., Grosveld F., and de Laat W. 2002. Looping and interaction between hypersensitive sites in the active beta-globin locus. Mol. Cell 10: 1453.

Verona R.I., Mann M.R., and Bartolomei M.S. 2003. Genomic imprinting: Intricacies of epigenetic regulation in clusters. Annu. Rev. Cell Dev. Biol. 19: 237.

Wakefield M.J., Keohane A.M., Turner B.M., and Graves J.A. 1997. Histone underacetylation is an ancient component of mammalian X chromosome inactivation. Proc. Natl. Acad. Sci. 94: 9665.

Weber M., Hagege H., Murrell A., Brunel C., Reik W., Cathala G., and Forne T. 2003. Genomic imprinting controls matrix attachment regions in the Igf2 gene. Mol. Cell. Biol. 23: 8953 .

Yoon B.J., Herman H., Sikora A., Smith L.T., Plass C., and Soloway P.D. 2002. Regulation of DNA methylation of Rasgrf1. Nat. Genet. 30: 92.

Yusufzai T.M. and Felsenfeld G. 2004. The 5'-HS4 chicken beta-globin insulator is a CTCF-dependent nuclear matrix-associated element. Proc. Natl. Acad. Sci. 101: 8620.

Yusufzai T.M., Tagami H., Nakatani Y., and Felsenfeld G. 2004. CTCF tethers an insulator to subnuclear sites, suggesting shared insulator mechanisms across species. Mol. Cell 13: 291. 


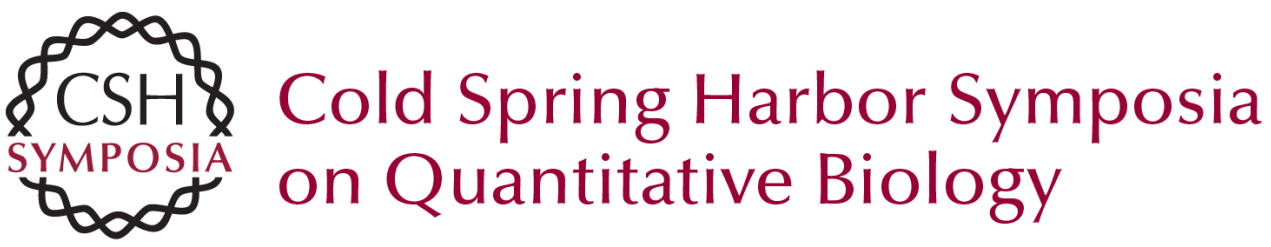

\section{Chromosome Loops, Insulators, and Histone Methylation: New Insights into Regulation of Imprinting in Clusters}

W. REIK, A. MURRELL, A. LEWIS, et al.

Cold Spring Harb Symp Quant Biol 2004 69: 29-38

Access the most recent version at doi:10.1101/sqb.2004.69.29

References This article cites 70 articles, 25 of which can be accessed free at: http://symposium.cshlp.org/content/69/29.full.html\#ref-list-1

\section{License}

Email Alerting

Receive free email alerts when new articles cite this article - sign up in Service the box at the top right corner of the article or click here. 